\title{
Follicular Ameloblastoma
}

\author{
Dr. Suraj Agarwal, MDS, \\ Oral Medicine \& Radiology \\ Director, Dantika Maxillofacial Telerad Centre \\ Ex. Post Graduate Student, ITS Dental College Hospital \& Research Centre, Greater Noida \\ *Corresponding Author: Dr. Suraj Agarwal, Oral Medicine \& Radiology, Director, Dantika \\ Maxillofacial Telerad Centre, Ex. Post Graduate Student, ITS Dental College Hospital \& Research \\ Centre, Greater Noida.
}

\section{CASE REPORT}

A 31 year old male patient reported to the department of oral medicine and radiology with a chief complain of pain and swelling in left lower back tooth region since 8 months. Patient gave history of trauma 8 months back, after which swelling developed which was pea size slowly increase in size over left side of face. Patient gave history of dull, intermittent pain which aggravates on taking food. Patient underwent FNAC and OPG 1 day back. Extra oral examination (Figure 1) revealed diffuse swelling on left side of face at ramus of mandible. Extension of swelling anteroposteriorly from a st. Line drawn to beyond posterior border of mandible.Supero-inferiorlyfrom a st. Line drawn from ala of nose to tragus $1 \mathrm{~cm}$ beyond inferior border of mandible. Overlying skin appears to be normal. Swelling soft to firm in consistency, tender on palpation with no rise in temperature. Lymph node examination- left Submandibular lymph node palpable, mobile, soft to firm in consistency \& tender on palpation. Intraoral examination - obliteration of buccal vestibule region w.r.t 37,38 with no expansion of buccal \& lingual cortical plate. Diffuse swelling extending over anterior border of ramus of mandible, slightly tender on palpation. No egg shell crackling present. Pulp vitality test - vital w.r.t 36,37, non-vital w.r.t 38. Clinical diagnosis of fracture of left side of mandible?, odontogenic cyst or tumor of mandible?. OPG (Figure 2) reveals a large multilocular lesion on left side of ramus of mandible exending to condylar process associated periapical to 37, 38. CBCT (Figure 3, 4, 5) reveals A large, expansile osteolytic lesion is noted in left body-ramus of mandible, extending from \#37 region till postero-inferior third of ramus \& cranially till sigmoid notch area. Expansion, thinning and intermittent effacement of adjoining cortical boundaries, loss of trabecular architecture is seen with appearance of multi-locularity due to parttern of trabecular destruction in cranio-caudal and mediolateral thirds of the lesion. Cortical effacement of left mental IAC \& indistinct left mandibular foramen. Incisional biopsy was performed and histopathologic (Figure 6) diagnosis of FOLLICULAR AMELOBLASTOMA was given.
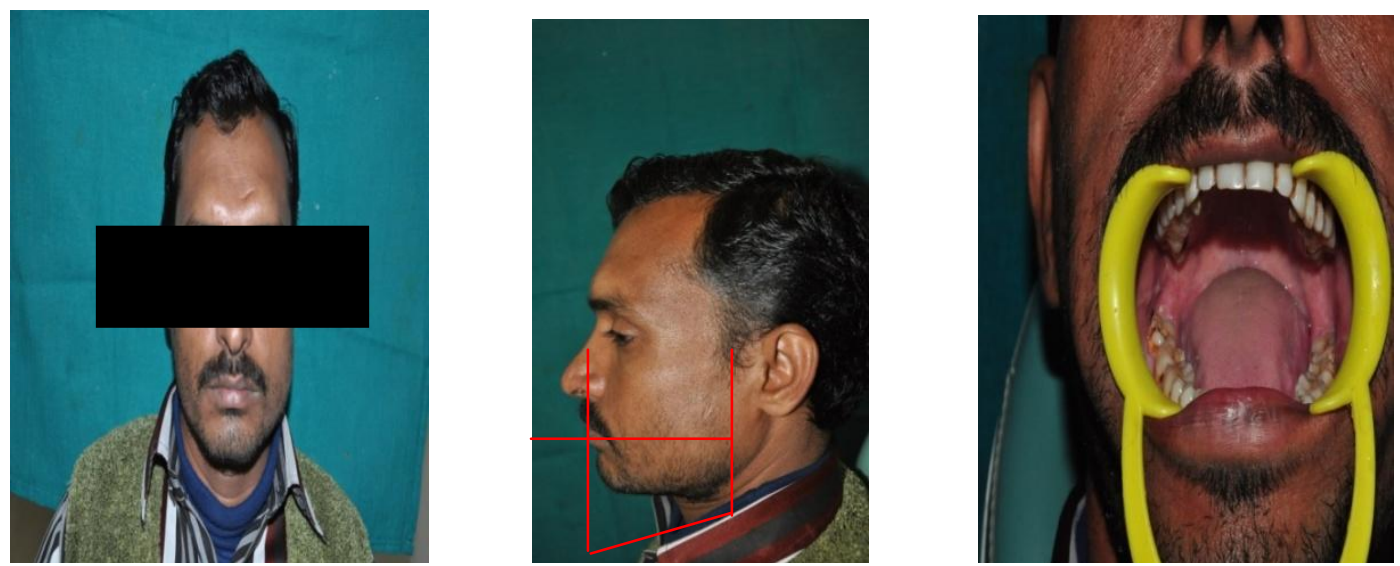

Figure1. Frontal \& Lateral Profile reveals facial asymmetry with diffuse swelling on left side of angle of mandible. 


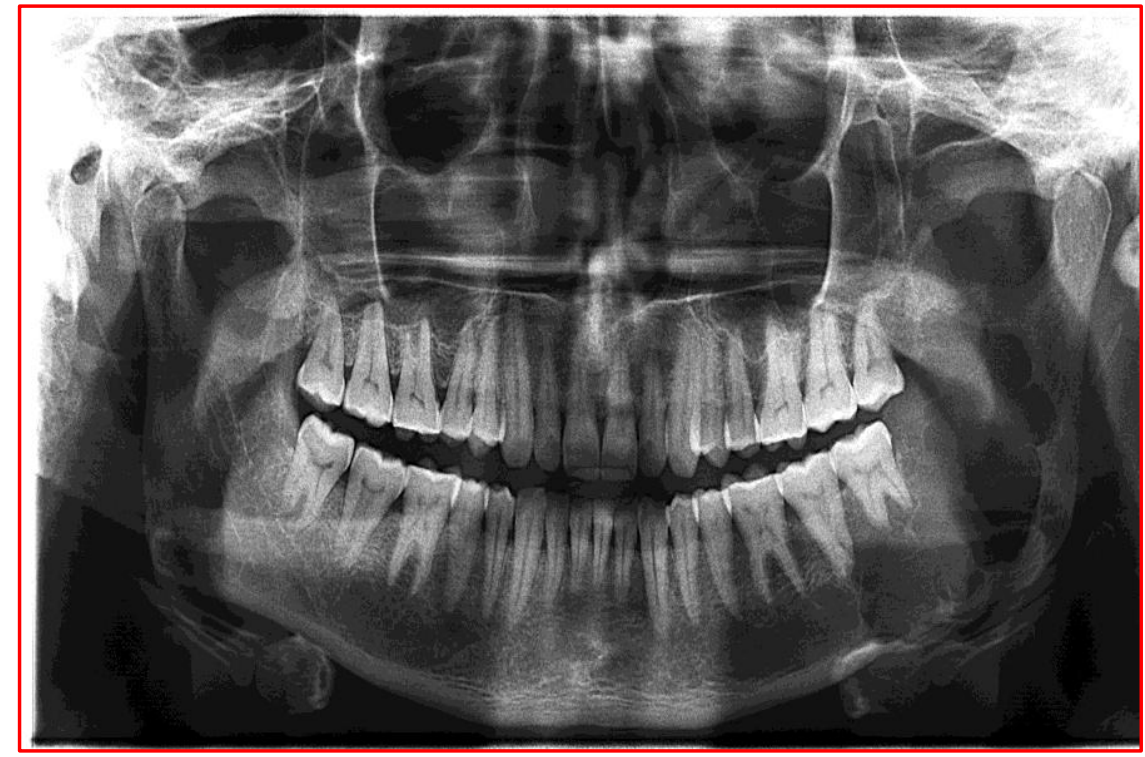

Figure2. OPG reveals a large multilocular lesion on left side of ramus of mandible exending to condylar process associated periapical to 37,38
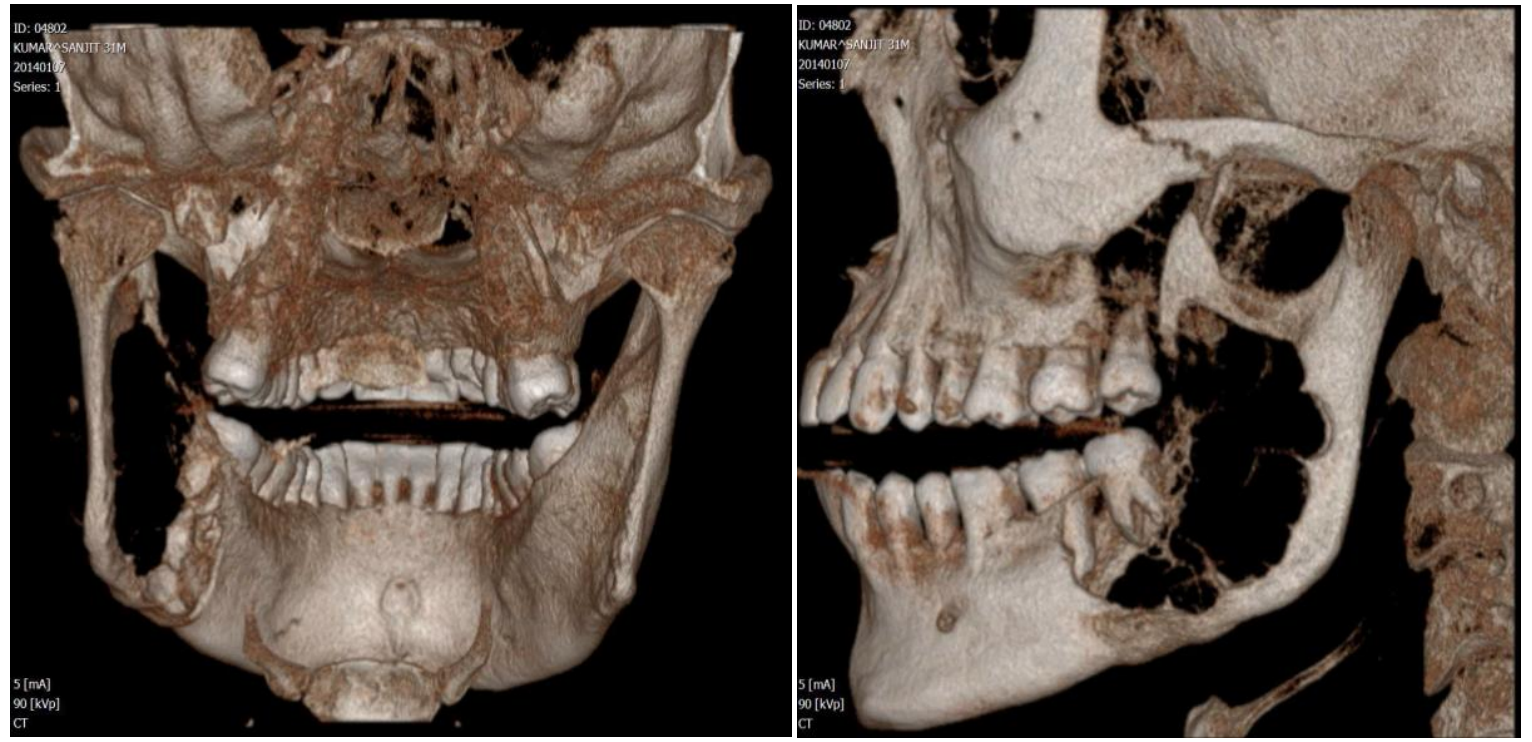

Figure3. $C B C T-3 D$ Sections

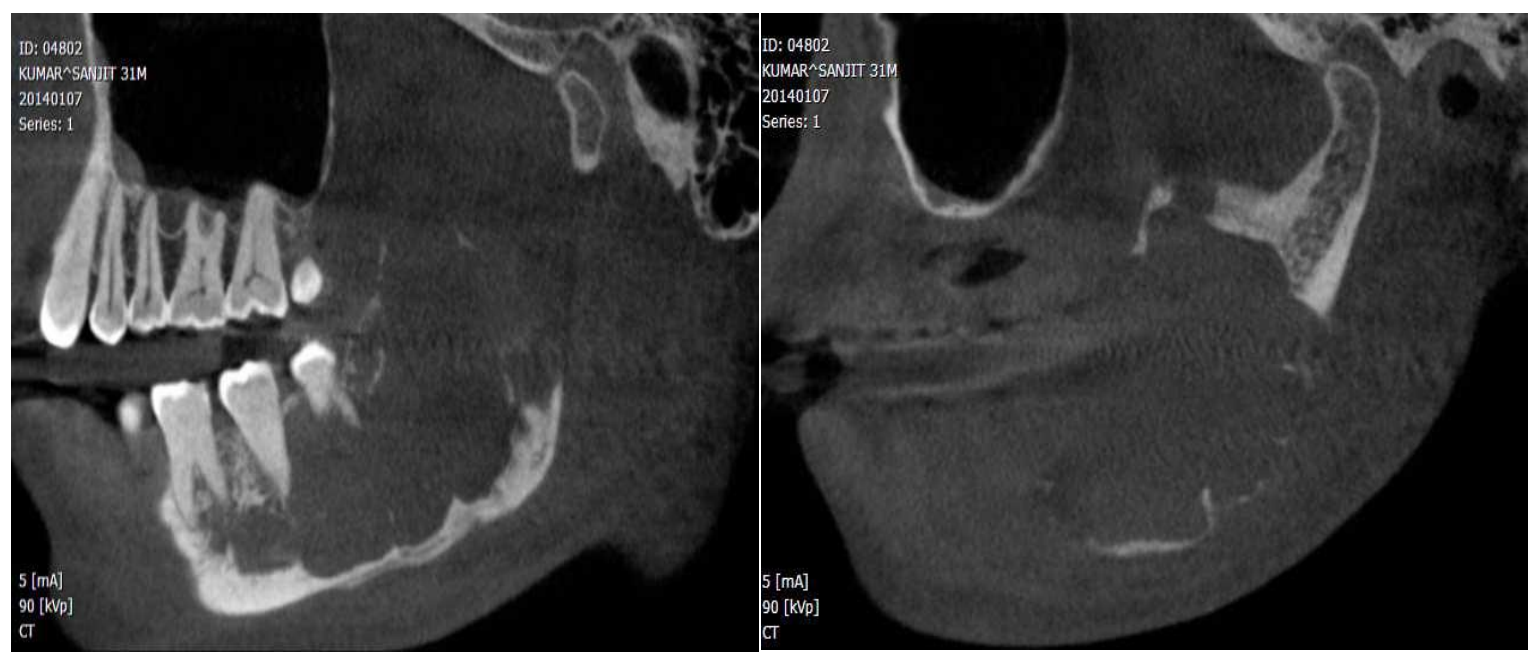

Figure4. CBCT-Sagittal Sections 


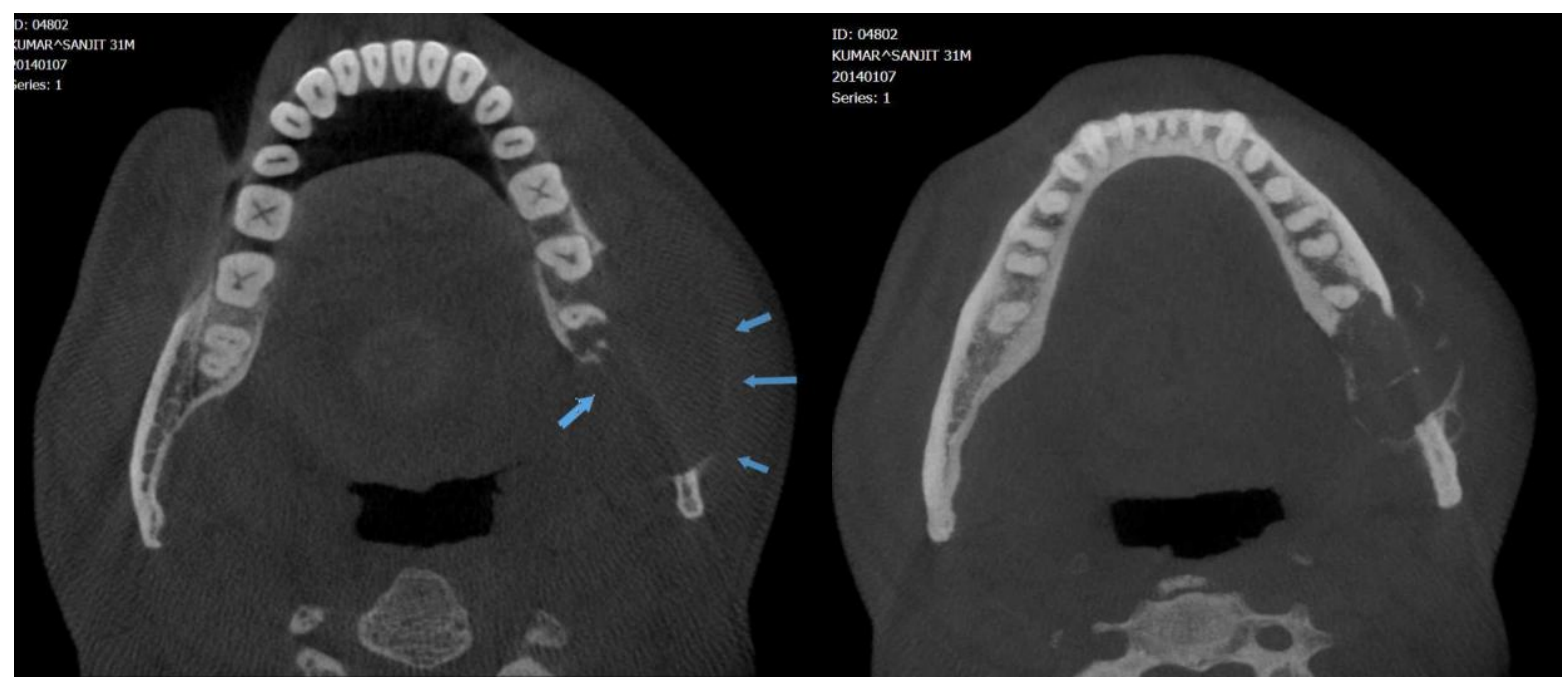

Figure5. $C B C T-$ Cross Sections

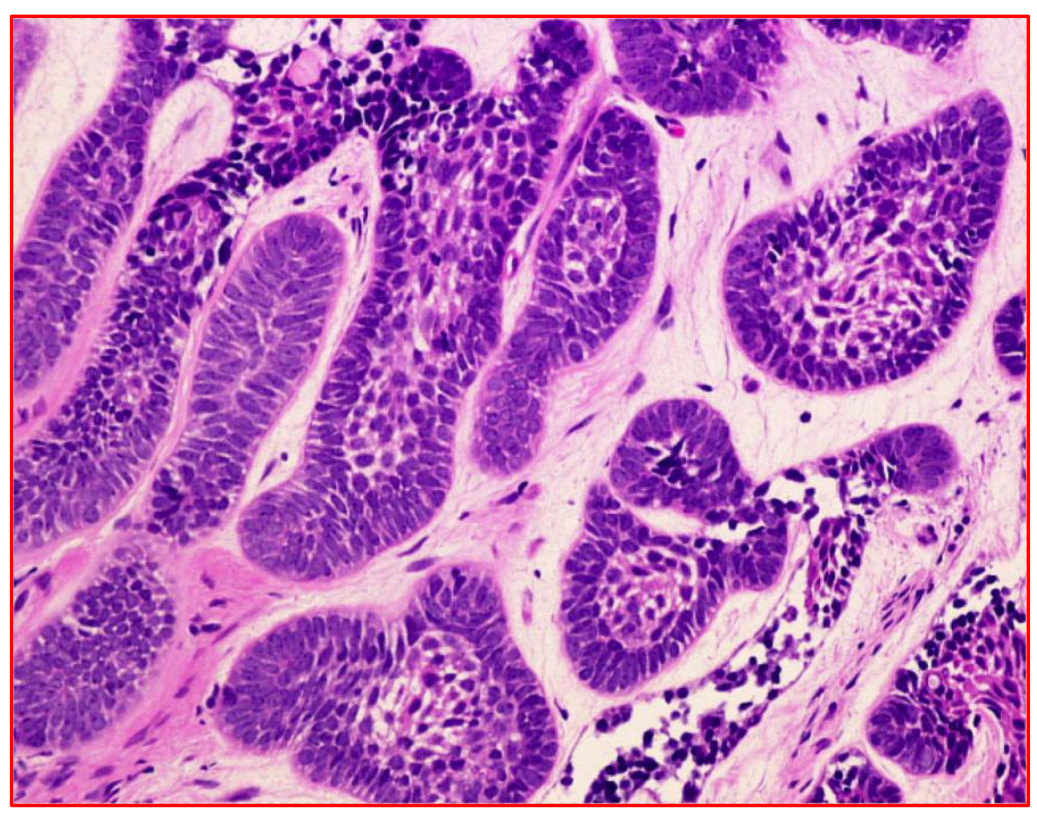

Figure6. Histopathology Image

Citation: Suraj A. Follicular Ameloblastoma. International Journal of Research Studies in Medical and Health Sciences. 2017;2(5):5-7.

Copyright: () 2017 Suraj A. This is an open-access article distributed under the terms of the Creative Commons Attribution License, which permits unrestricted use, distribution, and reproduction in any medium, provided the original author and source are credited. 